\title{
Intracluster entropy from quasar activity
}

\author{
A. Lapi and A. Cavaliere \\ Università "Tor Vergata", Via Ricerca Scientifica 1, I-00133 Roma, Italy
}

\begin{abstract}
We discuss how the energy feedback from active galactic nuclei and quasars is essential in explaining the observed X-ray luminosities and entropy levels of the intracluster and intragroup medium.
\end{abstract}

\section{Introduction}

Clusters, groups, and many single galaxies shine in X-rays because they contain large masses of hot plasma that radiate strong luminosities by thermal bremsstrahlung and line emission. Simple conditions are found to prevail in rich clusters; they emit copious X-ray powers $L_{X} \propto n^{2} R^{3} \sqrt{T} \sim 10^{45} \mathrm{erg} \mathrm{s}^{-1}$ integrated over sizes $R \sim$ Mpc. The temperatures $k T \gtrsim 4 \mathrm{keV}$ are close to the virial values $k T_{v} \propto G M / R$ in the gravitational potential wells provided by dark matter $(\mathrm{DM})$ masses $M \sim 10^{15} M_{\odot}$. The gas densities $n \sim 10^{-3}$ $\mathrm{cm}^{-3}$ are consistent with baryon to DM fractions $m / M \sim 1 / 5$, the universal value.

However, surprises arise in moving to groups and galaxies. Similarly simple conditions would imply the "gravitational" scaling $L_{X} \propto T^{2}$ (Kaiser 1986), or $L_{X} \propto T$ when lines become important. Instead, the luminosities, recently observed or revised, are lower by factors $10^{-1}-10^{-2}$, see Fig. 1 . This also shows how the emissions from small groups and galaxies scatter widely and often downward, a feature of non-Gaussian, largely intrinsic nature (Mushotzky 2004).

Thus in the smaller structures the plasma is clearly underdense for its observed temperatures. The issue is reinforced on considering that the specific entropy $s$ of the plasma (or rather the adiabat $K=k T / n^{2 / 3} \propto e^{2 s / 3 k}$, see Bower 1997) is enhanced relative to the scaling $K \propto T$ that is expected if the gas had been gravitationally heated by shocks in the cluster outskirts as it was accreted, see Fig. 2. This implies that extra energy inputs occurred during the formation of groups and clusters.

\section{External preheating from AGNs}

We focus on the substantial energies fed back to the gaseous baryons, when part of them condense into massive stars then exploding as type II supernovae (SNe), or accrete on to central supermassive black holes energizing active galactic nuclei (AGNs; see Valageas \& Silk 1999; Nath \& Roychowdhury 2002).

During the hierarchical buildup such energy inputs preheat the gas before it falls into new groups and clusters, so hindering its flow into the latter. In smaller systems the accretion shocks are increasingly modulated, thus inducing lower baryon densities and higher entropy levels relative to a pure gravitationally-driven infall (Lapi, Cavaliere \& Menci 2004).

But SNe provide only a mild preheating, around $1 / 4 \mathrm{keV}$ per particle; the resulting luminosity depressions or entropy enhancements are shown by the light strips in Figs. 1 and 2 to be limited both in amplitude and in scatter (see Cavaliere, Lapi \& Menci 2002).

Including the additional contribution of AGNs raises the preheating to $3 / 4 \mathrm{keV}$ per particle, if their energy outputs are coupled to the ambient baryons at fractional levels 


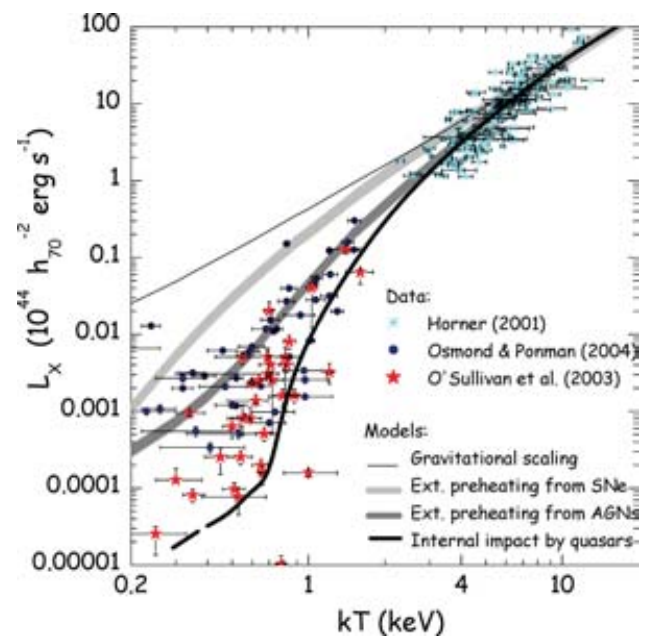

Figure 1. Integrated X-ray luminosity $L_{X}$ vs. X-ray temperature $T$.

$f \sim 5 \%$, as independently gauged by observations with CHANDRA and XMM-Newton (see Chartas, Brandt \& Gallagher 2003; Pounds et al. 2003). Such a preheating value provides a sizeable step toward the locus of the data, as shown in Figs. 1 and 2 by the heavy strips.

However, the observations in groups and galaxies are only marginally fitted, while the wide scatter in the $L_{X}-T$ correlation is still unaccounted for.

\section{Internal impact of quasars}

But right in groups and galaxies the impulsive inputs by powerful quasars have an additional impact from inside. This occurs when the energy $\Delta E \sim 2 \times 10^{62} f\left(M_{\bullet} / 10^{9} M_{\odot}\right)(1+$ $z)^{-3 / 2}$ ergs injected over $10^{8}$ yr for an accreted mass $M_{\bullet}$ competes with the value $E \sim$ $2 \times 10^{61}(k T / \mathrm{keV})^{5 / 2}(1+z)^{-3 / 2}$ ergs for the gas in equilibrium. The ratio $\Delta E / E \sim 0.5$ $(f / 5 \%)\left(M_{\bullet} / 10^{9} M_{\odot}\right)(k T / \mathrm{keV})^{-5 / 2}$ approaches unity in poor groups or galaxies. Then over $10^{2} \mathrm{pc}$ a quasar wind can act as an efficient piston to drive through the gas a blastwave terminated by a shock (Granato et al. 2004).

We derive (Lapi, Cavaliere \& De Zotti 2003) self-similar solutions for the blast flow perturbing an equilibrium plasma density $n(r) \propto r^{-2}$ (or steeper) under the push of the energy addition $\Delta E(t) \propto t$ (or flatter). These blasts also include the restraints set by a finite initial pressure and by DM gravity; still, the ratio of the kinetic to the thermal energy ranges from $10^{-1}$ up to 2 .

Thus within a dynamical time the blast ejects beyond the cluster outskirts a considerable gas mass $\Delta m / m \simeq \Delta E / 2 E$ (for $\Delta E / E \lesssim 2$ ), and cause an average temperature raise $\Delta T / T \simeq \Delta E / 10 E$ of the residual medium. In the equilibrium recovered after ejection, the X-ray luminosity $L_{X} \propto(1-\Delta m / m)^{2} T^{2}$ is depressed and the entropy $K \propto(1-\Delta m / m)^{-2 / 3} T$ enhanced relative to the initial values (see solid lines in Figs. 1 and 2). We find that the current $\mathrm{X}$-ray data require the diffuse baryons to absorb a fraction $f \sim 5 \%$ of the full quasar output. Furthermore, in terms of increasingly strong, sporadic quasar impacts we can understand why the intrinsic scatter seen in the data widens toward poor groups.

On approaching the galactic scales no further density depletion takes place (knee in the solid line of Fig. 1), because the internal feedback from quasars is self-regulated (Silk 


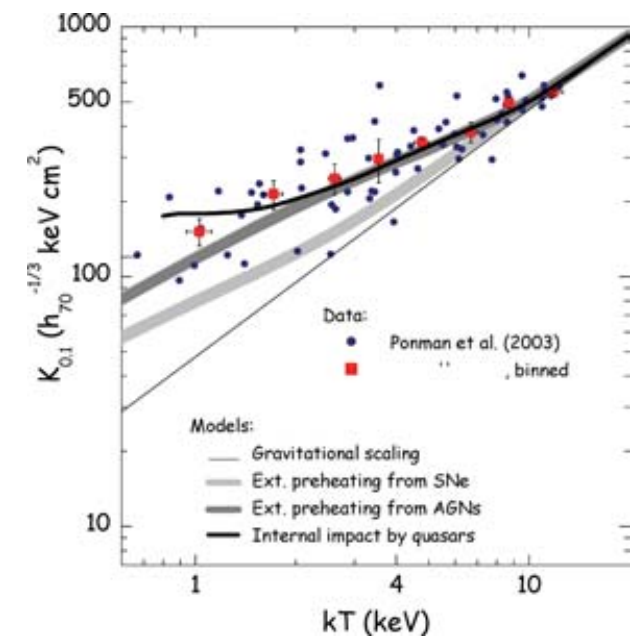

Figure 2. Central entropy $K$ vs. X-ray temperature $T$.

\& Rees 1998; King 2003). In other words, $\Delta E / E$ cannot exceed a few, lest the energizing accretion is stopped (Cavaliere et al. 2002). Pleasingly, with the same value $f \sim 5 \%$ indicated by the X-ray data, such a limiting condition yields the steep correlation $M_{\bullet} \approx$ $5 \times 10^{8} M_{\odot}(f / 5 \%)^{-1}\left(\sigma / 300 \mathrm{kms}^{-1}\right)^{5} \propto \sigma_{\star}^{4}$ observed in the optical band (Ferrarese 2002) between the black hole masses and the galaxian velocity dispersions. In the microwave and submm bands (Lapi et al. 2003) real-time evidence of quasar impact in action may be caught from Sunyaev-Zel'dovich signals enhanced by overpressure in running blastwaves.

\section{Acknowledgements}

We are grateful to N. Menci for stimulating comments, and to the organizers for an interesting Colloquium.

\section{References}

Bower, R.G. 1997 MNRAS 288, 355.

Cavaliere, A., Lapi, A., \& Menci, N. 2002 ApJ 581, L1.

Chartas, G., Brandt, W.N., \& Gallagher, S.C. 2003 ApJ 595, 85.

Ferrarese, L. 2002 ApJ 578, 90.

Granato, G.L., De Zotti, G., Silva, L., Bressan, A., \& Danese, L. 2004 ApJ 600, 580.

Horner, D.J. 2001 Ph.D. Thesis, Univ. of Maryland.

Kaiser, N. 1986 MNRAS 222, 323.

King, A.R. 2003 ApJ 596, L27.

Lapi, A., Cavaliere, A., \& De Zotti, G. 2003 ApJ 597, L93.

Lapi, A., Cavaliere, A., \& Menci, N. 2004 ApJ, submitted.

Mushotzky, R.F. 2004 Carnegie Observatories Astrophysics Series Vol. 3 (Cambridge: Cambridge Univ. Press).

Nath, B.B., \& Roychowdhury, S. 2002 MNRAS 333, 145.

Osmond, J.P.F., \& Ponman, T.J. 2004 MNRAS, submitted (preprint astro-ph/0402439).

O'Sullivan, S., Ponman, T.J., \& Collins, R.S. 2003 MNRAS 340, 1375.

Ponman, T.J., Sanderson, A.J.R., \& Finoguenov, A. 2003 MNRAS 343, 331.

Pounds, K.A., King, A.R., Page, K.L., \& O’Brien, P.T. 2003 MNRAS 346, 1025.

Silk, J., \& Rees, M.J. 1998 A\&JA 331, L1.

Valageas, P., \& Silk, S. 1999 A $\& A$ 350, 725. 